\title{
Design and Testing of a 2-DOF Flexure-based Compliant Stage
}

\author{
Yunsong $\mathrm{Du}^{1}$, Tiemin $\mathrm{Li}^{1}$, Wei $\mathrm{Ji}^{2}$ and Yao Jiang ${ }^{1}$ \\ ${ }^{1}$ Mechanical Engineering Department, Tsinghua University, 100084, Beijing, China \\ ${ }^{2}$ Foreign Language Department, Hebei Normal University for Nationalities, Chengde, China
}

\begin{abstract}
This paper presents the mechanical design and testing of a 2-DOF flexure-based compliant stage. The prismatic joints are first discussed. To obtain high resonance frequency and decoupled property, the stage composed of four parallel chains with symmetrical configuration should be employed. Finally, an experimental platform is set up to test the cooperative tracking performance. The experimental trajectory is in good agreement with the desired trajectory under various input frequencies. It shows that the maximum tracking error exhibits an increase from $0.8 \%$ to $2.7 \%$. The experiment results demonstrate that the proposed compliant stage possess good tracking performance under $100 \mathrm{~Hz}$, which also validates the reasonability of the stage.
\end{abstract}

\section{Introduction}

Flexure-based compliant mechanisms have many advantages, such as no clearance, no friction losses, no need for lubrication, compactness, and ease of fabrication [1-3]. Considering these advantages, they have been widely used in many industrial applications, such as scanning probe microscopy [4-6], micro-grippers [7-8], and biological science [9-10].

In the past decades, various flexure-based compliant stages have been developed. Awtar [11] presented a parallel XY flexure mechanism designs based on systematic constraint patterns. The advantages of the parallel flexure structure were discussed, and the constraint arrangement was given directly. Qin [12] designed a 2-degree of freedom (DOF) decoupled flexure-based mechanism which was composed of statically indeterminate symmetric flexure structures in parallel. The symmetric design helped to eliminate the thermal error and obtain decoupled property. The experiment results demonstrated that the proposed mechanism had good decoupled ability and load capability, and wide potentials in precision positioning applications. Yong [13] introduced a flexure-based XY stage for fast nanoscale positioning. The design of the flexure-based nanopositioning stage was based on the concept of flexible mechanisms. The stage was composed of two main parts: the outer section that consisted of amplification levers and circular flexures, and the inner section that included a stage and beam flexures. Li [14] designed a totally decoupled flexure-based XY parallel micromanipulator. The double compound parallelogram flexures and a compact displacement amplifier were employed, and they were simple and symmetric. Both finite element analysis and experiments could validate the decoupled property.
There have been many compliant stages with various good performances, such as decoupled ability, high resonance frequency and large workspace. Nevertheless, the design of these existing mechanisms is not systematic. Therefore, the present study addresses the design and testing of a 2-DOF flexure-based mechanism. The mechanical design is first introduced, and the experiments on cooperative tracking performance are tested. The remaining sections of this paper are organized as follows. In section 2, the mechanical design of the stage is derived. In section 3 , a prototype of the stage is manufactured an experimental platform is set up. The circular tracking performance is subsequently tested under various input frequencies (from 20 to $100 \mathrm{~Hz}$ ).

\section{Mechanical design}

Flexure-based mechanisms can be classed into two types: planar and space mechanisms. The planar flexure-based mechanisms have been applied most widely, and they are usually composed of several prismatic joints, such as linear, parallelogram and amplifying prismatic joints. A linear prismatic joint is illustrated in Fig. 1, and it is composed of flexure hinges, rigid beams and one output platform. The structure is symmetric about the output platform and both ends are fixed. Being symmetric, the output platform would rotate around the center point $O$ when a moment $M_{z}$ is applied. In addition, the output platform can translate along $y$-axis if the force $F_{y}$ is applied to point $O$. The parasitic motions in $x$-axis and the parasitic rotations about $z$-axis can be attenuated by the symmetrical configuration. By contrast, it could remain static in conjunction with consideration of the high stiffness along $x$-axis when the force $F_{x}$ is applied to point $O$. 


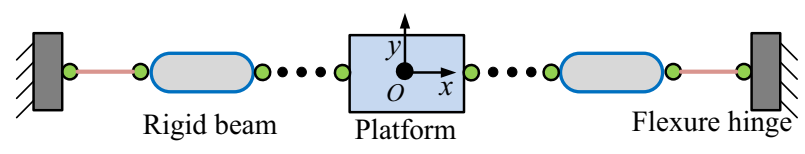

Figure 1. The linear prismatic joint

The parallelogram and double parallelogram prismatic joints are the common joints which can provide translational motion. To derive the decoupled property, flexure-based mechanisms are usually composed of double parallelogram rather than parallelogram. As shown in Fig. 2, the double parallelogram is composed of four limbs, and both sides of the joint are fixed.

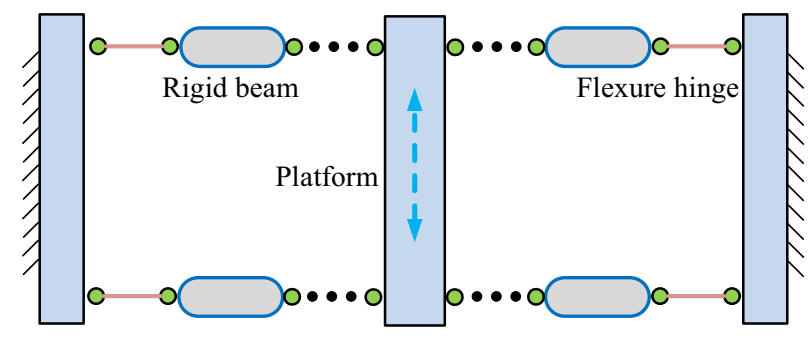

Figure 2. The double parallelogram prismatic joint

For a notch flexure hinge, there are strong couplings between the rotational and translational motions. Furthermore, the rotation center of a flexure hinge drifts whenever the hinge works, resulting in motion errors. To obtain decoupling properties, multiple flexure hinges can be combined to form certain structures. As illustrated in Fig. 3, the parallelogram prismatic joint can keep the orientation of the output platform invariant when the platform is actuated by the force $F_{x}$ along $x$-axis. However, undesired cross-coupling error $e_{y}$ along $y$-axis is generated at the same time. Compared with the parallelogram joint on one side, with a symmetrical configuration about the actuating axis, the double parallelogram prismatic joint can eliminate the coupling error completely, and thus it possesses output decouple property. In practice, the parallelogram joints are usually used as prismatic joints.
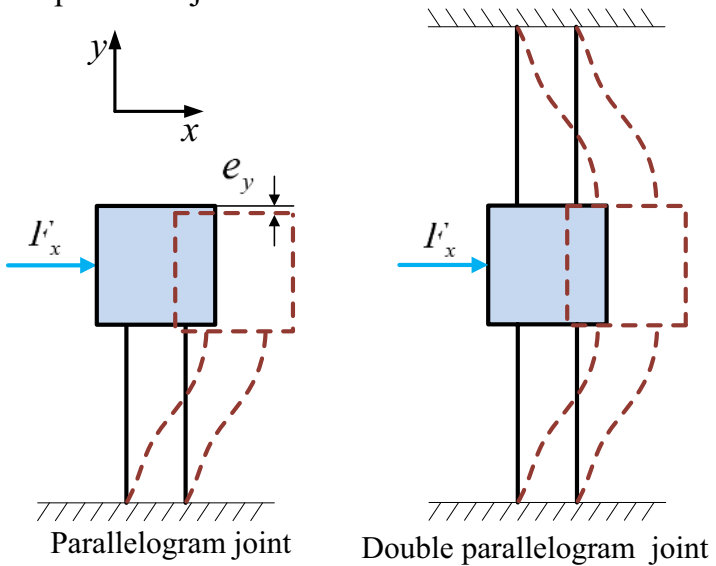

Figure 3. Parallelogram and double parallelogram joint

The amplifying prismatic joint is similar to the bridge-type displacement amplification mechanism. As shown in Fig. 4, surface $D$ is fixed. There is a displacement output on surface $\mathrm{C}$ when imposing a force on surfaces $A$ and B. Using this joint, the input displacement can be amplified by more than ten times. Nevertheless, it is only used for compliant mechanisms with low resonant frequency.

In general, flexure-based mechanisms can be classified into two categories according to the kinematic structure: the serial and parallel mechanisms. Serial mechanisms are easier to design, and they have substantially decoupled degree-of-freedom. By contrast, parallel mechanisms with closed-loop kinematic features possess many advantages, such as high load carrying capacity, rigidity and accuracy.

According to the kinematic structure, the flexurebased micro-motion stages can be classified into two categories: the serial structure and the parallel structure. The parallel structure which possesses high rigidity, high load carrying capacity, and high accuracy could be selected. To derive high resonant frequency, the parallel structure should be employed, and the stages should be rigid and compact.

Flexure-based parallel mechanisms are usually built on 2-PP、3-RRR $、 3-P P P$ and 6-SPS parallel structure, where $P, R, U$, and $S$ denote the revolute, prismatic, universal and spherical joints, respectively. To achieve 2DOF motion, the 2-PP parallel structure could be selected. In addition, to achieve high precise motion, magnetic levitation motors, electromagnetic actuators, voice coil motors and piezoelectric actuators (PZTs) are usually employed. The PZTs are selected to drive the flexure-based compliant stage because they can provide fast and ultra-precision motions.

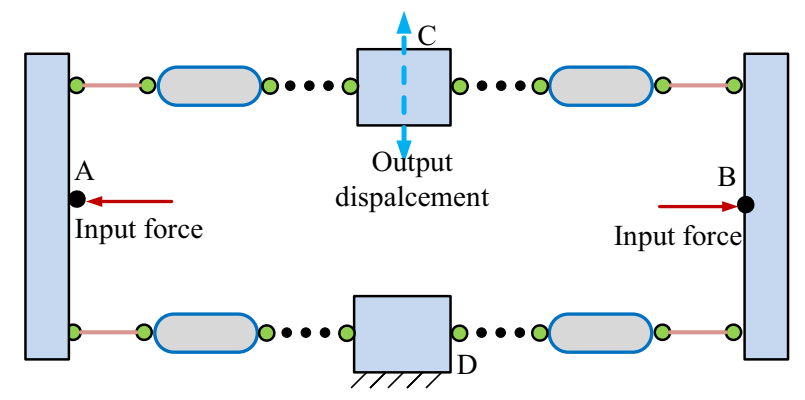

Figure 4. The amplifying prismatic joint

Meanwhile, to obtain decoupled property, the stage with symmetrical configuration should be employed, and thus it can be depicted as Fig. 5. It is composed of four parallel chains, and each chain consists of two prismatic joints. One joint $P_{1}$ is used to provide the forces actuated by the PZTs to the stage. The other joint $P_{2}$ is employed to transmit the forces and deformations of the flexure hinges to the output platform.

According to the analysis above, to obtain compact structure, $P_{1}$ and $P_{2}$ can be the linear and parallelogram prismatic joints rather than the amplifying prismatic joints. In addition, the parallelogram is virtually composed of several linear prismatic joints. Therefore, only the linear prismatic joints could be employed to form the stage. 


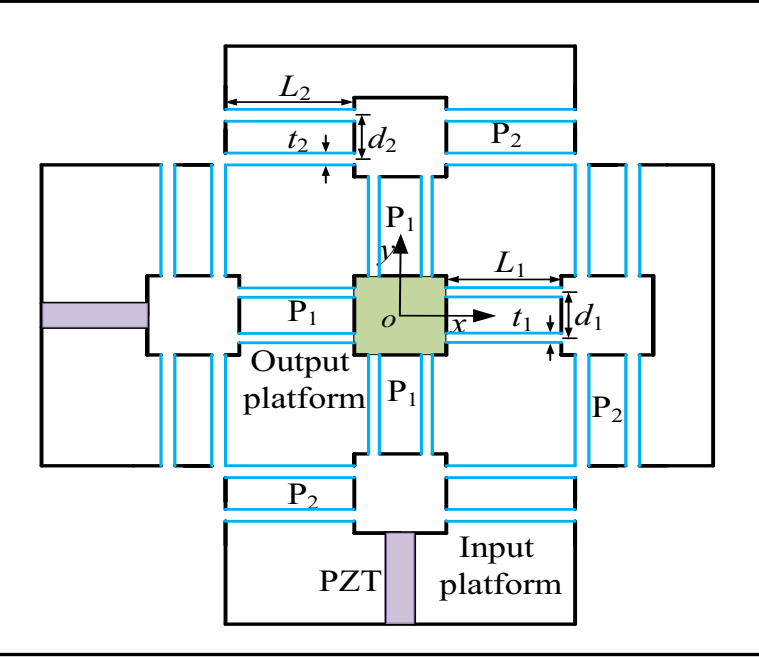

Figure 5. The flexure-based compliant stage

Notch flexure hinges can be manufactured by drilling or milling to form a circular, corner-filled or constant rectangular cutout. A flexure hinge is actually a complex spring element that can respond to and transmit both rotation and translation. The constant rectangular crosssection flexure hinge is selected because it is simple and easy to design. The prismatic joints $P_{1}$ and $P_{2}$ are composed of several flexure hinges, and their performances are easily influenced by their geometrical parameters. Thus, the parameters of the stage should be optimized.

The objective of the optimization is to maximize the resonance frequency. Meanwhile, the parameters are optimized to obtain the workspace of the stage which is above $18 \mu \mathrm{m}$. The thickness of the stage is chosen as 10 $\mathrm{mm}$ to guarantee the stiffness of the stage in $\mathrm{z}$-axis. Meanwhile, the length and width of the output platform could be selected as $20 \mathrm{~mm} \times 20 \mathrm{~mm}$ to install sensors and accessories. Considering the size of the PZTs, the input platform is chosen as $20 \mathrm{~mm} \times 20 \mathrm{~mm}$ to connect to the mechanism and PZTs. The flexure hinge thickness $t$ can be divided into $n$ parts. According to the analysis in our earlier paper [15], the divided parts $n$ are selected 2 to maximize the resonance frequency, and the optimized parameters are listed as follows: $L_{1}=25 \mathrm{~mm}, L_{2}=28 \mathrm{~mm}$, $t_{1}=2.5 \mathrm{~mm}, t_{2}=3 \mathrm{~mm}, d_{1}=11.5 \mathrm{~mm}$ and $d_{2}=11 \mathrm{~mm}$.

\section{Experiments}

An experimental platform is set up. It includes a dSPACE-DS1107 board, two catalog-length gauges (MT 1281), two PZTs (PSt 150/7/40 VS12 from XMT Harbin, Inc.), a piezo amplifier and drive (XE500/E05 from XMT Harbin, Inc.), and the proposed flexure-based compliant stage. As illustrated in Fig. 6, the material of the stage is aluminum 7075. The two preloaded PZTs are actuated by the driver under control of the dSPACE board, with a voltage range of $0-120 \mathrm{~V}$. To realize the PI control, the displacements of the output platform are measured by two catalog-length guages (MT 1281 from Heidenhain, Inc.). To reduce vibrations, the air spring optical platform (1209M-100T) made in South Korea is employed.

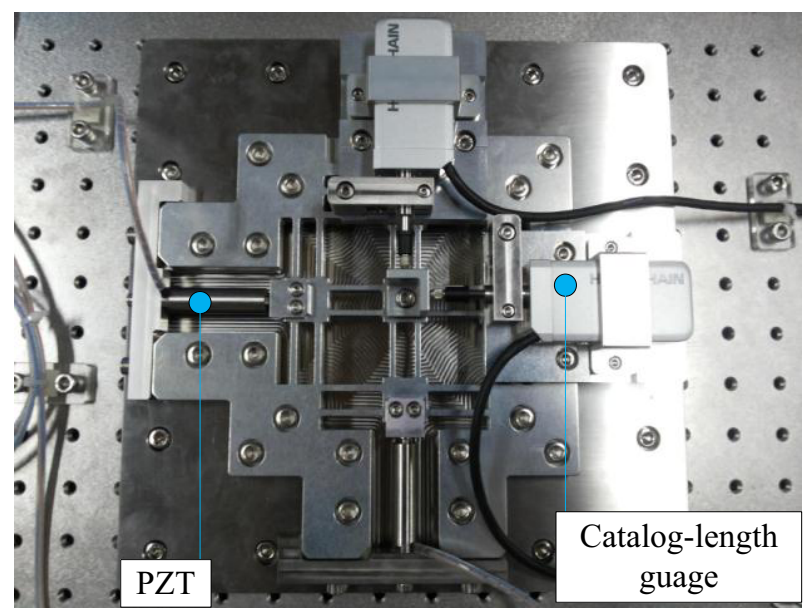

Figure 6. The experiment platform

The dSPACE board can be applied for receiving displacement feedback from the catalog-length gauges, calculating the control, and then sending the it to the amplifier and drive. The 16-bit analog to digital converters is used to translate the analog voltage positional signal to digital signals used by the PI controller. The amplifier and drive can be empoyed to receive command signals from the PI controller and provide amplified voltage to drive the preloaded PZTs.

To verify the proposed mechanical design of the compliant stage, the cooperative tracking performance is tested. A circular trajectory test is performed under different frequencies (from $20 \mathrm{~Hz}$ to $100 \mathrm{~Hz}$ ), and the input circular radius is $5 \mu \mathrm{m}$. The compliant stage can be actuated by driving it in $x$-axis and $y$-axis with the appropriate amplitudes and phase quadrature (shifted in phase by 90 degrees). Without loss of generality, only the circular tracking performance test under input frequency $80 \mathrm{~Hz}$ is illustrated in Fig. 7. It can be seen that the actual trajectory derived from experiments is in good agreement with the desired trajectory. The tacking error is less than $100 \mathrm{~nm}$, and the maximum error is $2 \%$ in terms of the circular radius $5 \mu \mathrm{m}$.

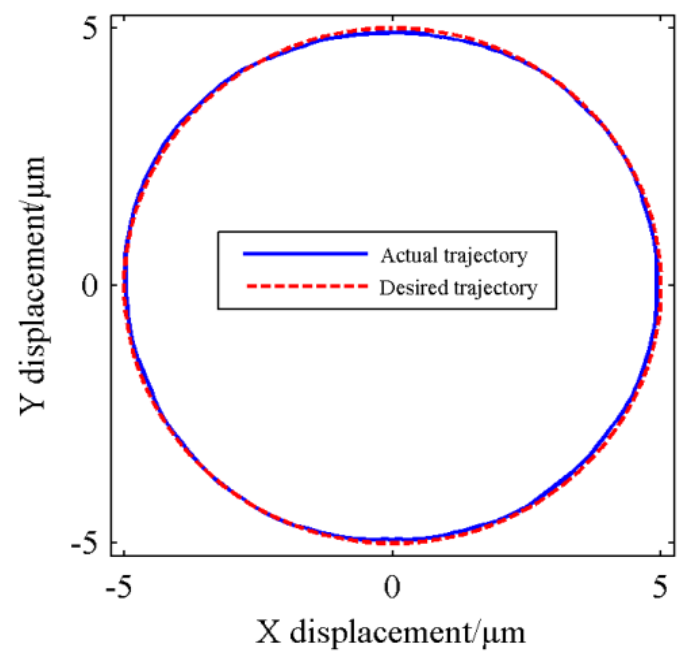

(a) Tracking results 


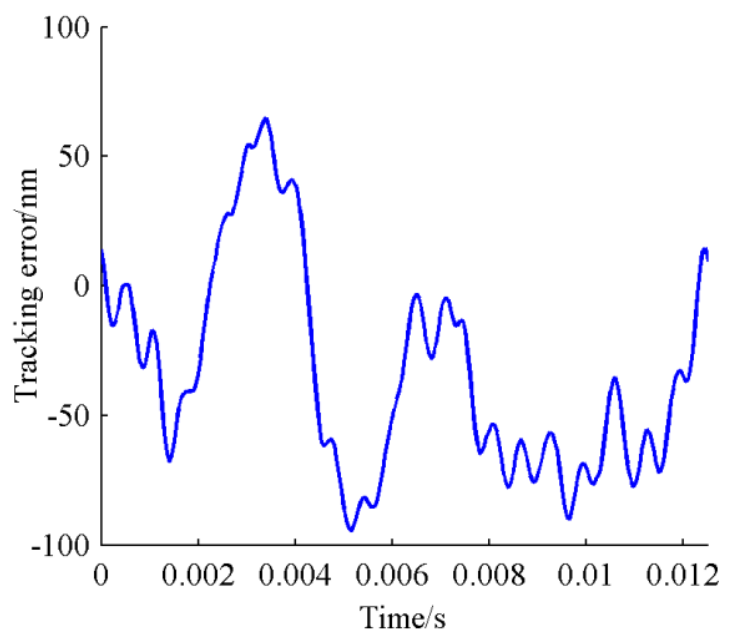

(b) Tracking errors

Figure 7. Circular tracking performance under $80 \mathrm{~Hz}$

The circular tracking error under various input frequencies is studied, as demonstrated in Fig. 8. The tracking error exhibits an increase with increasing the input frequency. To be more specific, the maximum tracking exhibits an increase from $0.04 \mu \mathrm{m}$ to $0.135 \mu \mathrm{m}$. Considering that the input circular radius is $5 \mu \mathrm{m}$, the maximum tracking error would be $2.7 \%$ under input frequency $100 \mathrm{~Hz}$, and the minimum tracking error would be $0.8 \%$ under input frequency $20 \mathrm{~Hz}$. Especially, the minimum tracking error would be $0.8 \%$ under input frequency $60 \mathrm{~Hz}$. The experiment results indicate that the flexure-based compliant stage possess good cooperative tracking performances.

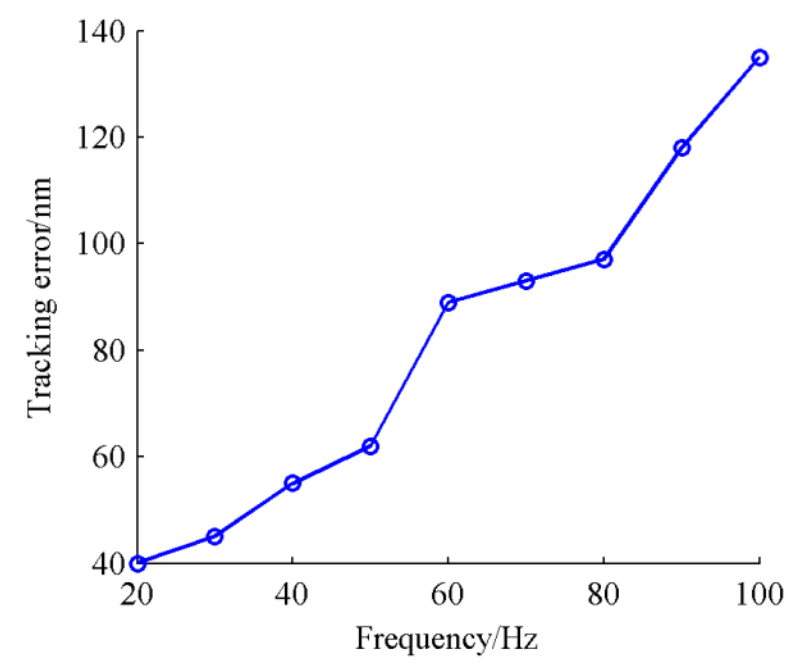

Figure 8. Tracking error under various input frequencies

\section{Conclusions}

This paper presented the mechanical design and testing of a flexure-based compliant stage with symmetrical configuration. The stage was designed based on the prismatic joints to obtain high resonance frequency and good decoupled property. The flexure parallel structure was selected, and it was composed of four parallel chains. Each chain consisted of two prismatic joints which were composed of the constant rectangular cross-section flexure hinges. The optimization is carried on to maximize the first resonance frequency. Finally, a prototype of the stage is manufactured and an experimental platform is set up. And the circular tracking performance was subsequently tested. The experiment results indicated that the proposed flexure-based compliant stage possessed good tracking performance (from 20 to $100 \mathrm{~Hz}$ ), which validates the reasonability of the design.

\section{Acknowledgement}

This work was supported by the National Basic Research Program of China (Grant No.2011CB302400), the National Science Foundation of China (Grant No.51275260), and the National Science and Technology Major Project of China (Grant No.2015ZX04001002).

\section{References}

[1] N. Lobontiu . Compliant mechanisms: design of flexure hinges. CRC press, 2010, pp.2-15.

[2] W. Widhiada. T. G. T. Nindhia, N. Budiarsa. Robust control for the motion five fingered robot gripper. . Int. J. Mech. Eng. And Rob. Res, 4 (2015), 226-232.

[3] Y. Tian, B. Shirinzadeh, D. Zhang. Three flexure hinges for compliant mechanism designs based on dimensionless graph analysis. Precision Engineering, 34 (2010), 92-100.

[4] G. Schitter, K. J. Åström, B. E. DeMartini, P. J. Thurner, K. L. Turner, P. K. Hansma. Design and modeling of a high-speed AFM-scanner. IEEE. T. CONTR. SYST. T, 15 (2007), 906-915.

[5] B Sollapur Shrishail, P Deshmukh Suhas. XY scanning mechanism: a dynamic approach. Int. J. Mech. Eng. And Rob. Res, 3 (2014), 140-154.

[6] Leang, K. K., and Fleming, A. J.: High - speed serial kinematic SPM scanner: design and drive considerations, Asian. J. Control, 11 (2009), 144-153.

[7] M. N. M. Zubir, B. Shirinzadeh, Y. Tian. Development of a novel flexure-based microgripper for high precision micro-object manipulation. Sensors and Actuators A: Physical, 150 (2009), 257-266.

[8] Q. Xu. Design and development of a flexure-based dualstage nanopositioning system with minimum interference behavior. IEEE. T. Autom. Sci. Eng, 9 (2012), 554-563.

[9] T. Ando, N. Kodera, D. Maruyama, E. Takai, K. Saito, A. Toda. A high-speed atomic force microscope for studying biological macromolecules in action, Jpn. J. Appl. Phys, 41 (2002), 4851

[10] H. Y. Kim, D. H. Ahn, D. G. Gweon. Development of a novel 3 degrees of freedom flexure based positioning system, Rev. Sci. Instrum, 83 (2012), 055114.

[11] S. Awtar, A. H. Slocum. Constraint-based design of parallel kinematic XY flexure mechanisms. J. Mech. Des, 129 (2007), 816-830.

[12] Y. Qin, Y. Tian, D. Zhang. Design and dynamic modeling of a 2-DOF decoupled flexure-based mechanism. Chin. J. Mech. Eng, 25 (2012), 688-696.

[13] Y. K. Yong, S. S. Aphale, S. O. R. Moheimani. Design, identification, and control of a flexure-based XY stage for fast nanoscale positioning. IEEE. T. Nanotechnol, 8 (2009), 46-54. 
[14] Li Y, Xu Q. Design and analysis of a totally decoupled flexure-based XY parallel micromanipulator. IEEE. T. Robot, 25 (2009), 645-657.
[15] Y. S. Du, T. M. Li, Y. Jiang, H. T. Wang. Design and analysis of a 2-degree-of-freedom flexure-based micromotion stage. Adv. Mech. Eng. 8 (2016), 1-13. 